\title{
Sustained Vascular Endothelial Growth Factor Delivery Enhances Angiogenesis and Perfusion in Ischemic Hind Limb
}

\author{
Qinghua Sun, ${ }^{1}$ Ruth R. Chen, ${ }^{2}$ Yuechun Shen, ${ }^{1}$ David J. Mooney, ${ }^{2}$ Sanjay Rajagopalan, ${ }^{1}$ and \\ P. Michael Grossman ${ }^{1,3}$
}

Received January 9, 2005; accepted April 27, 2005

Purpose. We hypothesized that sustained delivery of vascular endothelial growth factor (VEGF) using a polymer [85:15 poly(lactide-co-glycolide) (PLG)] would enhance angiogenesis and improve perfusion of ischemic tissue.

Methods. C57BL/6J mice ( $n=20 /$ group) underwent unilateral hind limb ischemia surgery and were randomized to groups of no scaffold implantation ( $\emptyset$-Implant), unloaded scaffold implantation (EmptyPLG), or implantation of scaffolds incorporating $3 \mu \mathrm{g}$ of $\mathrm{VEGF}_{165}$ (PLG-VEGF). Endpoints included laser Doppler perfusion imaging (LDPI, ischemic/nonischemic limb, \%), local vessel counts, immunohistochemistry for CD31, and $\alpha$-smooth muscle actin. In vitro release kinetics of VEGF from PLG was also measured.

Results. PLG-VEGF resulted in improved lower extremity perfusion $v s$. controls as measured by LDPI $\%$ at $7,14,21$, and 28 days $(p<0.05)$. PLG-VEGF was associated with significantly greater percentage of vessels staining for CD31 and $\alpha$-smooth muscle actin compared to the Empty-PLG or $\emptyset$-Implant $(p<0.05$ for both)

Conclusions. The PLG-VEGF scaffolds resulted in sustained VEGF delivery, improved tissue perfusion, greater capillary density, and more mature vasculature compared to the controls. The sustained-release PLG polymer vehicle is a promising delivery system for therapeutic neovascularization applications.

KEY WORDS: angiogenesis; ischemia; polymer; vascular endothelial growth factor.

\section{INTRODUCTION}

There exists a critical need for new therapies to treat ischemic peripheral arterial disease (PAD). Strategies designed to enhance lower extremity blood flow via therapeutic angiogenesis/arteriogenesis are attractive modalities because they address the fundamental issue of limited blood flow in PAD (1). Preclinical and clinical efforts to accomplish the latter goal have focused on systemic delivery of proangiogenic growth factors or regional delivery approaches using gene transfer at the site of ischemia (2). Whereas several growth factors, including vascular endothelial growth factor

\footnotetext{
${ }^{1}$ Division of Cardiovascular Medicine, Department of Internal Medicine, University of Michigan Hospitals and Health System, Ann Arbor, Michigan 48109-0311, USA

${ }^{2}$ Department of Biologic and Materials Sciences, University of Michigan, Ann Arbor, Michigan 48109, USA

${ }^{3}$ To whom correspondence should be addressed. (e-mail: pagross@ umich.edu)

ABBREVIATIONS: Empty-PLG, PLG polymer not loaded with growth factor; $\emptyset$-Implant, hind limb surgery performed with no PLG polymer implanted; PAD, peripheral arterial disease; PLG, poly(lactide-co-glycolide); PLG-VEGF, PLG polymer loaded with $3 \mu \mathrm{g}$ of $\mathrm{VEGF}_{165}$; SMA, smooth muscle actin; VEGF, vascular endothelial growth factor.
}

(VEGF), initiate new blood vessel growth, protracted expression of VEGF may be required for activation of endothelial and hematopoietic progenitor cells (3) and for maintenance of stable neovessels (4). Current gene transfer approaches with viral or plasmid vectors have limitations related to their duration of expression-often less than 3 weeks (5). This may represent one reason underlying the failure of human clinical trials with gene transfer approaches for patients with PAD (6) and has led to an interest in techniques designed to increase the local concentration, time of growth factor exposure, or both. A polymer-based drug delivery vehicle has several potential advantages including protracted and localized drug delivery (7-9). One promising drug delivery system utilizes a biodegradable polymer of lactide and glycolide [poly(lactide-co-glycolide), PLG] (10-12). This polymer was developed as a biodegradable suture (13) and has a long history of use in humans. The many advantageous features of PLG (e.g., controlled degradation, well-established tissue interaction, etc.) make it an attractive vehicle for drug delivery. The rationale underlying this approach is that the bioactive molecule, e.g., VEGF, is incorporated into the polymer and then implanted at the site of desired administration. The bioactive factor is slowly and controllably released from the polymer (e.g., via polymer degradation), with the dose and rate of delivery dependent on the amount of drug loaded, the process used for drug 


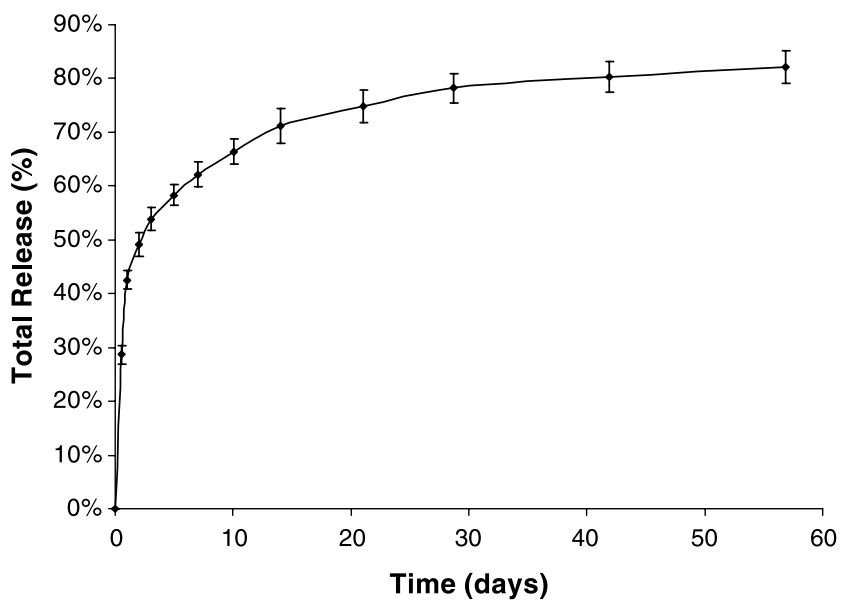

Fig. 1. In vitro cumulative release kinetics of VEGF from scaffolds fabricated from PLG (85:15, lactide/glycolide), measured using ${ }^{125} \mathrm{I}$-labeled tracers. Data represent the mean $(n=5)$, and error bars represent standard deviation.

incorporation, and the polymer used to fabricate the vehicle (14). VEGF delivery with various PLG vehicles has been previously reported to enhance local neovascularization $(7,12,15,16)$, but the functional impact of this vascularization on tissue perfusion has not been reported.

In this study, we hypothesized that sustained delivery of VEGF using a degradable polymer scaffold (85:15 PLG) incorporating human $\mathrm{VEGF}_{165}$ would result in development of mature vasculature and improvements in perfusion in a murine model of hind limb ischemia. A mouse hind limb ischemia model was used in this study. Endpoints included tissue perfusion with laser Doppler, immunohistochemical indicators for neovascularization that included the endothelial cell marker CD31 (17) and $\alpha$-smooth muscle actin $(\alpha$ SMA) (18), which is expressed in both pericytes and smooth muscle cells and is associated with mature blood vessels.

\section{MATERIALS AND METHODS}

\section{Animals and Surgical Procedures}

Sixty male C57BL/6J mice (Jackson Laboratories, Bar Harbor, ME, USA) aged 6 weeks were used in these studies. Animals were handled according to a protocol approved by the University of Michigan Committee on Use and Care of Animals. Mice were cared for according to standards in the Guide for the Care and Use of Laboratory Animals. Before the surgical procedures, the mice were anesthetized with an intraperitoneal injection of a mixture of ketamine $80 \mathrm{mg} / \mathrm{kg}$ and xylazine $5 \mathrm{mg} / \mathrm{kg}$. Severe unilateral hind limb ischemia was surgically created via ligation and division of the left superficial femoral artery and vein, left external iliac artery and vein, and left deep femoral and circumflex arteries and veins. The consistency of limb ischemia and the prognosis associated with this model was previously confirmed in our laboratory (data not shown). At the time of surgery, mice were randomized to one of three groups ( $n=20$ per group): immediate implantation of PLG scaffold containing VEGF
(PLG-VEGF), PLG scaffold containing no growth factor (Empty-PLG), or no PLG scaffold ( $\emptyset$-Implant). The scaffolds were implanted in the thigh region adjacent to the ligated vessels.

\section{Scaffold Fabrication and Analysis of Release Kinetics in Vitro}

Porous polymer scaffolds capable of VEGF release in a sustained pattern were fabricated from PLG using a variation of a high-pressure carbon dioxide fabrication process as previously described (15). Briefly, all scaffolds were formed by an identical process of mixing PLG in the form of microspheres (particle size $5-50 \mu \mathrm{m}$ in diameter), $\pm 4 \mu \mathrm{g}$ of human $\mathrm{VEGF}_{165}$ lyophilized with alginate and sodium chloride particles. The inclusion of alginate has been previously demonstrated to increase the efficiency of protein incorporation into the PLG vehicle (7). The VEGF incorporation efficiency has been previously measured for this system $(7,15)$, and the amount of VEGF used to form scaffolds results in $3 \mu \mathrm{g}$ of VEGF in each scaffold at the end of the fabrication process. The PLG, $\mathrm{VEGF}_{165}$, and sodium chloride were mixed, compressed, and equilibrated with high-pressure carbon dioxide. When the pressure was released, the PLG particles expanded into the spaces between the salt particles and fused, entrapping VEGF and the salt. Subsequently, the salt particles were removed by leaching with water to yield porous scaffolds $(14,15,19)$. Scaffolds for in vitro release kinetics studies were fabricated with $3 \mu \mathrm{g}$ of ${ }^{125} \mathrm{I}$ VEGF tracer using an identical process, and the in vitro release studies were performed as previously described (15). In the release studies, the amount of VEGF incorporated into each scaffold is measured before starting the study (using the known specific activity of the iodinated VEGF), and release is normalized to the total VEGF incorporated into each scaffold. All scaffolds were $4.2 \mathrm{~mm}$ in diameter by $2.3 \mathrm{~mm}$ thick.

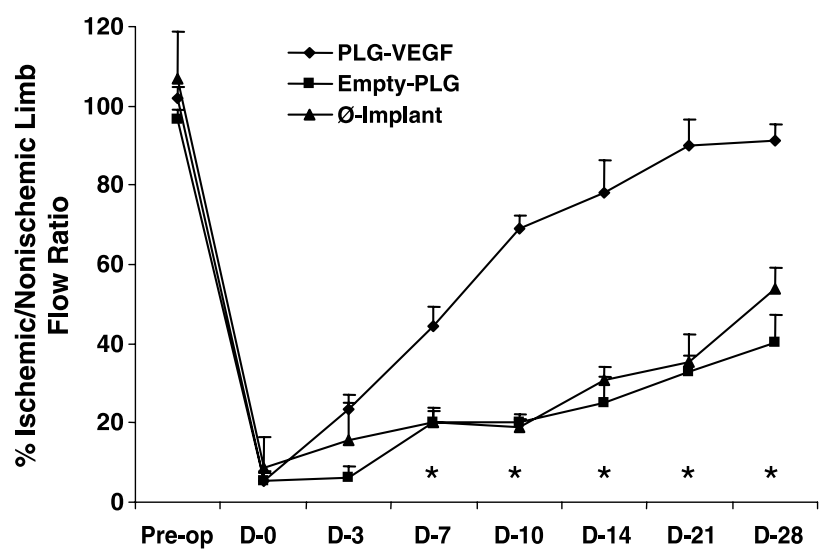

Fig. 2. Computer-assisted quantitative analyses of LDPI indices of hind limb blood flow in C57BL/6J mice. Blood flow was expressed as ischemic limb/untreated limb perfusion in mice implanted with PLGVEGF scaffolds compared with mice implanted with blank scaffolds or mice with sham surgery (no scaffold implantation). $*, p<0.05$, PLG-VEGF $v s$. Empty-PLG or $\emptyset$-Implant scaffold, $n=3-5$ at each time point and each group. 


\section{Laser Doppler Perfusion Imaging}

Hind limb blood flow was measured using a laser Doppler perfusion imaging (LDPI) system (Lisca Inc., Linköping, Sweden). Consecutive perfusion measurements were obtained by scanning the region of interest (hind leg and foot) of anesthetized animals. To account for variables that may affect blood flow temporally, the results at any given time were expressed against simultaneously obtained perfusion measurements as a ratio, i.e., left (ischemic)/right (normal) limb perfusion (20).

\section{Histological and Immunohistochemical Analysis}

Mice from each group were sacrificed at postsurgical days $7,14,21$, and 28 , and muscle and residual scaffolds were retrieved from the ischemic hind limb of each animal. The samples were fixed in $10 \%$ zinc formalin overnight, paraffin embedded, and sectioned into 5 - $\mu$ m-thick sections that were then stained with hematoxylin and eosin for histological analysis. Positive CD31 staining was visualized using a rat monoclonal antimouse platelet-endothelial cell adhesion molecule (PECAM)-1 antibody (CD31, 1:250, Pharmingen, San Diego, CA. USA), a biotinylated rabbit antirat $\mathrm{IgG}$ secondary antibody (1:200, Vector Laboratories, Burlingame, CA, USA), and NEN TSA Biotin System (NEL 700. Perkin Elmer Life Sciences, Boston, MA, USA) was used to visualize the bound antibodies. Omission of the primary antibody and replacement of the primary antibody by nonimmune $\mathrm{IgG}$ were used as a negative control for immunohistochemistry. The $\alpha$-SMA staining was performed using the Mouse on Mouse (MOM) Peroxidase Immunodetection kit (Vector Laboratories), primary antibody of mouse antihuman (1:50, Dako Cytomation, Carpinteria, CA, USA) and MOM secondary antibody.

Vessel densities calculated from slides stained for CD31 were expressed as capillaries per millimeter squared. To obtain the average vessel number per cross-sectional area, a

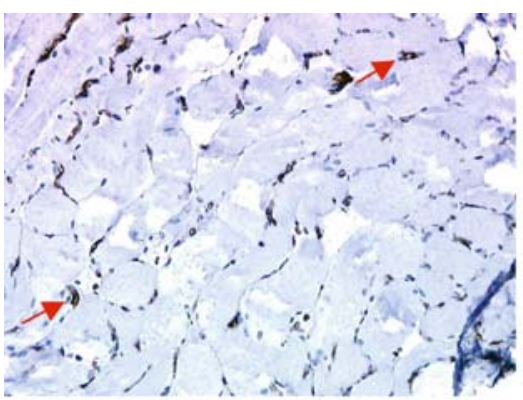

A-1. Ø-Implant

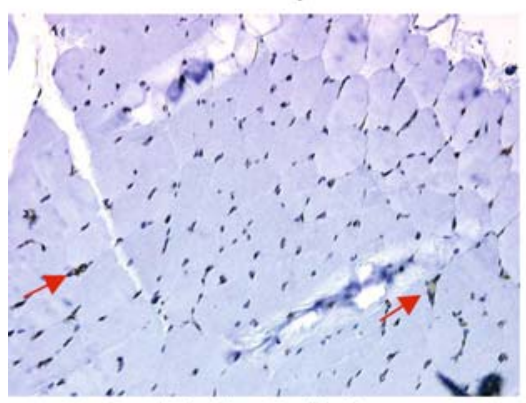

A-2. Empty-PLG

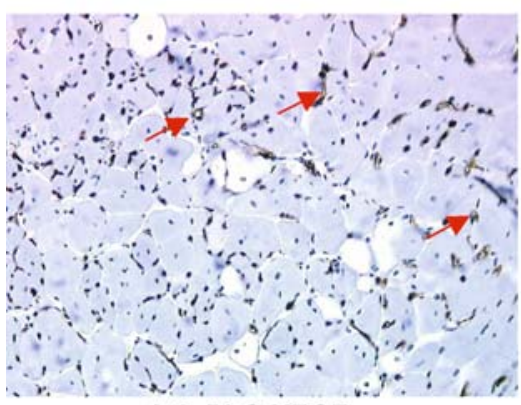

A-3. PLG-VEGF

Fig. 3. Representative images of CD31 expression from thigh muscle of C57BL/6J mice treated by Empty-PLG (A-2), PLG-VEGF (A-3), or hind limb ischemia surgery with no PLG scaffold implanted ( $\emptyset$-Implant) (A-1); and from retrieved scaffolds from Empty-PLG (B-1) and PLG-VEGF scaffold (B-2) at day 28. There are more darkstaining CD31 positive capillary vessels (arrows) in the PLG-VEGF group than in the Empty-PLG scaffold or $\emptyset$-Implant groups. Magnification for all photomicrographs was $\times 200$.

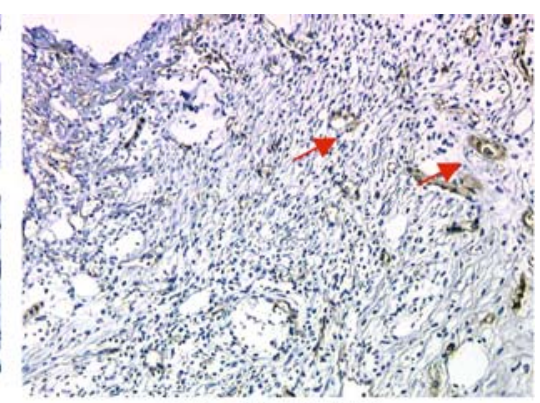

\section{B-1. Empty-PLG}

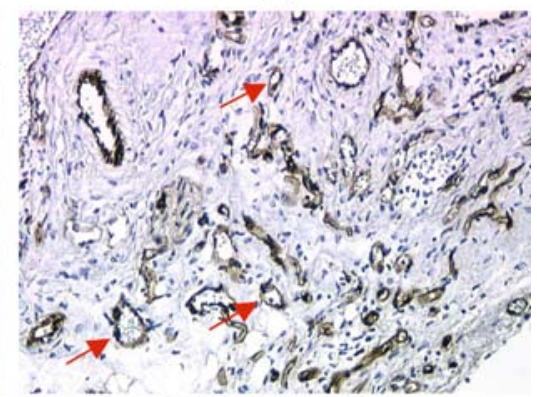

B-2. PLG-VEGF 
minimum of ten individual fields per slide were sampled, and Image Pro-plus 4.0 (Media Cybernetics, Silver Spring, MD, USA) was used to measure the counted field area. The number of CD31 positively stained blood vessels was counted manually. Only those CD31 positive vessels with a morphological circumference of one or greater endothelial cells were tallied as capillary vessels. CD31 staining consisting of a single cell was not counted as a capillary. The number of vessels associated with $\alpha$-SMA positively stained cells was also quantified in a similar fashion.

\section{Materials and Chemicals}

PLG (85:15, intrinsic viscosity $=1.5 \mathrm{dL} / \mathrm{g})$ was purchased from Alkermes (Cambridge, MA, USA). High molecular weight alginate (MVM) was obtained from ProNova (Oslo, Norway). VEGF 165 was purchased from Intergen (Purchase, NY, USA). Radiolabeled ${ }^{125}$ I-VEGF was purchased from Perkin Elmer Life Sciences (Boston, MA, USA).

\section{Statistical Analysis}

All values were expressed as mean \pm SD, and were analyzed by two-way ANOVA with post hoc Friedman test using GraphPad Prism software (version 3.00, GraphPad

A CD-31 stain from muscle tissue

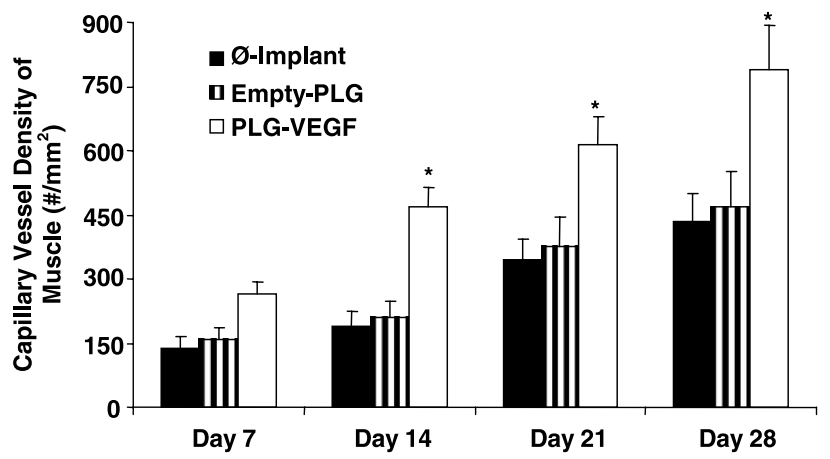

B CD-31 stain from retrieved PLG scaffold

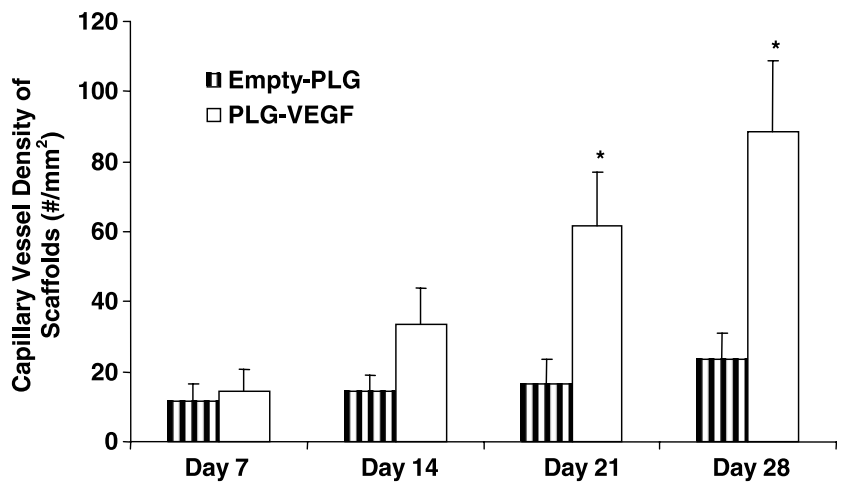

Fig. 4. Capillary densities after CD31 stain in thigh muscle (A) or retrieved scaffolds (B) of mice. There was significantly greater capillary density per square millimeter of ischemic tissue observed in mice treated by PLG-VEGF implantation vs. Empty-PLG or $\emptyset$ Implant implantation at postoperative days 14, 21, and 28. Similarly, there were significantly more capillary vessels in the retrieved scaffolds from the PLG-VEGF group compared to the retrieved EmptyPLG scaffolds at days 21 and 28 . Mean \pm SD $(* p<0.05)$.
Software, Inc., San Diego, CA, USA). A $p$ value $<0.05$ was considered significant.

\section{RESULTS}

\section{In Vitro Kinetics of VEGF Release}

Figure 1 demonstrates the in vitro kinetics of VEGF release in the scaffolds as assessed using ${ }^{125}$ I-VEGF. Approximately $50 \%$ of the VEGF was released from the PLG scaffold within 3 days. Subsequently, over the next 30 days, the VEGF release rate was approximately $1-3 \%$ per day.

\section{Lower Extremity Perfusion with VEGF Delivery via Biodegradable Scaffolds}

Figure 2 illustrates the LDPI index data in the three groups. In all groups, LDPI indices decreased immediately after surgery. In the $\emptyset$-Implant and Empty-PLG groups, the LDPI index remained $<20 \%$ for 10 days and then gradually increased to $42 \pm 6$ and $54 \pm 4 \%$, respectively by day 28 ( $p=$ ns). In contrast, the LDPI index in the PLG-VEGF group increased over time, was significantly greater than the other groups by day 7 , and was persistently greater than the other groups throughout the remainder of the study $(p<0.05$ for PLG-VEGF vs. other groups on days 7, 14, 21, and 28).

\section{Indices of Angiogenesis in Response to VEGF Scaffold Implantation}

Blood vessels were quantified in both the ischemic muscle tissue and within the space defined by the PLG delivery vehicles. The latter measurement reflects only the angiogenic response, as any vessels within the PLG must be newly formed. In contrast, both angiogenesis and arteriogenesis may be occurring in the muscle tissue. Muscle tissue capillary vessel density was significantly greater in the PLGVEGF group compared to the Empty-PLG or $\emptyset$-Implant group on days 14,21 , and 28 ( $p<0.05$ for PLG-VEGF $v s$. other groups) (Figs. 3 and $4 \mathrm{~A}$ ). In addition, there were significantly more capillary vessels in the retrieved scaffolds on days 21 and 28 ( $p<0.05$ for PLG-VEGF $v s$. other groups) (Figs. 3 and 4B). PLG-VEGF scaffold implantation was also associated with increased $\alpha$-SMA staining in the thigh muscle tissue compared to Empty-PLG and $\emptyset$-Implant $(p<0.05$ for PLG-VEGF vs. other groups) (Figs. 5 and 6). Similarly, vessels in the PLG-VEGF retrieved scaffolds had significantly more $\alpha$-SMA staining compared to the Empty-PLG scaffold group (Figs. 5 and 6).

\section{DISCUSSION}

The main findings of this study are the following. (1) Sustained growth factor presentation is feasible in the lower extremity using a biodegradable platform. (2) Protracted local presentation of VEGF is paralleled by improved lower extremity perfusion, greater capillary density, and more mature vasculature formation compared to the controls. These data support the concept of using polymer drug 


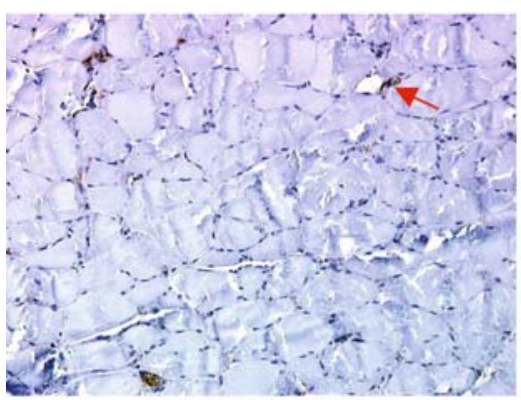

A-1. Ø-Implant

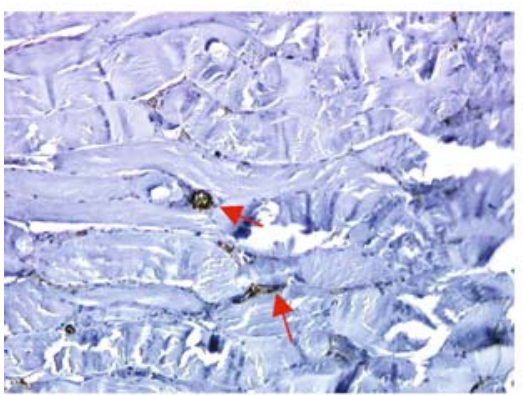

A-2. Empty-PLG

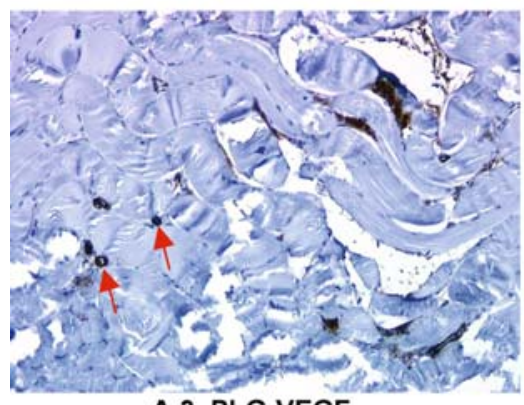

A-3. PLG-VEGF

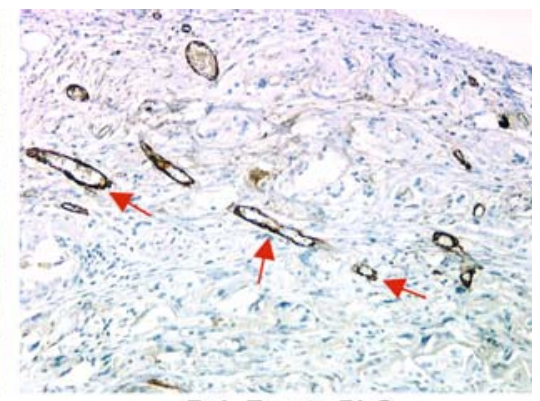

B-1. Empty-PLG

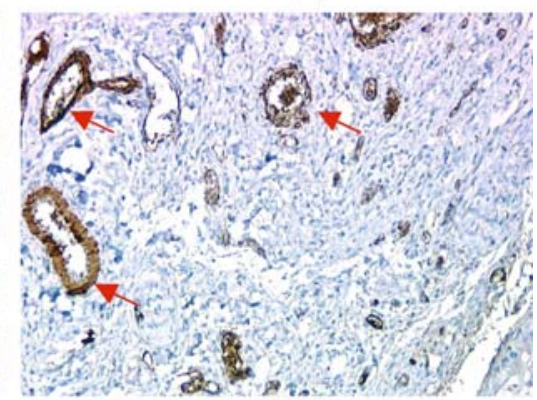

B-2. PLG-VEGF

Fig. 5. Representative images of $\alpha$-SMA staining of ischemic limb thigh muscle tissue of mice subjected to hind limb ischemia surgery without scaffold implantation $(\emptyset$ Implant) (A-1), Empty-PLG (A-2), PLG-VEGF (A-3) and from retrieved scaffolds from Empty-PLG (B-1) and PLG-VEGF (B-2) at day 28. There are more $\alpha$-SMA positive-staining vessels (arrows) in the PLG-VEGF group than in Empty-PLG or $\emptyset$ Implant group. Magnification for all photomicrographs was $\times 200$.

delivery to allow controlled sustained release of a bioactive factor (or factors). This study confirms and extends previous observations regarding VEGF delivery that have demonstrated improved indices of perfusion in the lower extremity with bolus delivery (21-24). However, this new delivery platform affords the opportunity to evaluate the effect of more protracted release of VEGF. This is especially important in light of observations that seem to suggest that the duration of exposure to VEGF may be a critical determinant of formation of stable neovasculature $(25,26)$.

The PLG vehicle used in this study has several potential advantages for delivery of therapeutic angiogenesis agents. The PLG polymer is biocompatible, degradable (27), and has been widely used in tissue engineering applications $(7,12,14,28-33)$. Because the growth factor release rate and tissue dose can be altered by adjusting polymer vehicle formulation $(19,27)$, the ability to influence local pharmacokinetics of the growth factor is substantial, and desired neovascular effects are achievable. The in vitro kinetics of VEGF release from the PLG system was similar to those previously reported with this approach to protein incorpora- tion $(12,14,15)$, but the different scaffold sizes, compositions, and physical forms of PLG used to fabricate the scaffolds (microparticles vs. preformed microspheres) in the various studies all can influence the release kinetics achievable with this system. Although not evaluated in this study, it has previously been demonstrated that VEGF incorporated and released over a 1-month period using this delivery system maintains a high level of bioactivity (15).

For this proof of concept investigation, VEGF was chosen because it has been demonstrated to promote neovascularization in preclinical studies $(6,34,35)$. VEGF is a multifunctional cytokine with potent vascular permeabilizing properties that stimulates endothelial cell migration, survival, and growth, and its effects on angiogenesis may be via direct action on endothelial cells and less direct effects involving other cells types in addition (36). In this study, PLG-VEGF-treated mice had increased hind limb blood flow, increased capillary density, and a sustained local tissue concentration of VEGF detectable for 14 to 28 days (data not shown). Previous studies with a PLG-VEGF scaffold manufactured with the technique identical to that used in 


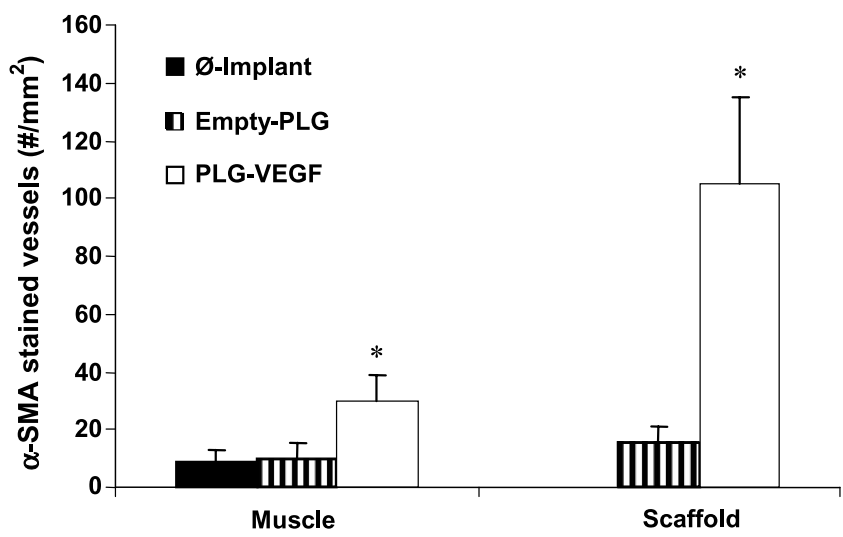

Fig. 6. $\alpha$-SMA positive-staining vessels from the muscle tissue and PLG scaffolds at day 28 . There were significantly more $\alpha$-SMA positive-staining vessels in both the PLG-VEGF group muscle tissue $(p<0.05)$ and PLG-VEGF group retrieved scaffold $(p<0.05)$. Mean \pm SD $(* p<0.05)$.

this study have confirmed that protein activity is maintained during scaffold fabrication and over a 1-month release period (15). The previous study also demonstrated that bolus delivery of an amount of VEGF equal to that incorporated into the PLG delivery vehicle led to no significant increase in capillary density, likely due to the extremely short half-life of this molecule when introduced unprotected into the body (15).

The PLG-VEGF group was associated with more mature blood vessels as determined by $\alpha$-SMA staining. This effect was seen in the muscle tissue, where this effect could be related to angiogenesis or arteriogenic alterations of preexisting vessels. However, the same effect was also noted within the space defined by the scaffold, in which all blood vessels must result from angiogenesis, indicating that the angiogenic process is being regulated by sustained VEGF delivery with this system. Interestingly, the relative diameter of vessels staining positive for $\alpha$-SMA was not significantly different among groups after 4 weeks (data not shown). This suggests that VEGF alone may be insufficient to induce vessel enlargement under the conditions of this study, and the action of additional factors such as platelet-derived growth factor may be required (15). Whether longer-term exposure to VEGF or combinations with other cytokine(s) would result in larger, more arteriolar appearing vasculature in ischemic tissue is under investigation.

\section{CONCLUSION}

Sustained VEGF delivery using the PLG polymer system results in improved tissue perfusion, greater capillary density, and more mature-appearing vasculature compared to the controls. These data extend previous in vitro and in vivo studies and suggest that this polymer system is a promising delivery approach for therapeutic angiogenesis and arteriogenesis.

\section{ACKNOWLEDGEMENT}

This work was financially supported by the National Heart, Lung and Blood Institute (R01 HL069957).

\section{REFERENCES}

1. J. M. Isnerand and T. Asahara. Angiogenesis and vasculogenesis as therapeutic strategies for postnatal neovascularization. J. Clin. Invest. 103:1231-1236 (1999).

2. M. Simons, R. O. Bonow, N. A. Chronos, D. J. Cohen, F. J. Giordano, H. K. Hammond, R. J. Laham, W. Li, M. Pike, F. W. Sellke, T. J. Stegmann, J. E. Udelson, and T. K. Rosengart. Clinical trials in coronary angiogenesis: issues, problems, consensus: an expert panel summary. Circulation 102: E73-E86 (2000).

3. S. Rafii, S. Meeus, S. Dias, K. Hattori, B. Heissig, S. Shmelkov, D. Rafii, and D. Lyden. Contribution of marrow-derived progenitors to vascular and cardiac regeneration. Semin. Cell Dev. Biol. 13: 61-67 (2002).

4. J. A. Nagy, E. Vasile, D. Feng, C. Sundberg, L. F. Brown, M. J. Detmar, J. A. Lawitts, L. Benjamin, X. Tan, E. J. Manseau, A. M. Dvorak, and H. F. Dvorak. Vascular permeability factor/ vascular endothelial growth factor induces lymphangiogenesis as well as angiogenesis. J. Exp. Med. 196:1497-1506 (2002).

5. H. K. Hammondand and M. D. McKirnan. Angiogenic gene therapy for heart disease: a review of animal studies and clinical trials. Cardiovasc. Res. 49:561-567 (2001).

6. S. Rajagopalan, E. R. Mohler, III, R. J. Lederman, F. O. Mendelsohn, J. F. Saucedo, C. K. Goldman, J. Blebea, J. Macko, P. D. Kessler, H. S. Rasmussen, and B. H. Annex. Regional angiogenesis with vascular endothelial growth factor in peripheral arterial disease: a phase II randomized, double-blind, controlled study of adenoviral delivery of vascular endothelial growth factor 121 in patients with disabling intermittent claudication. Circulation 108:1933-1938 (2003).

7. M. C. Peters, P. J. Polverini, and D. J. Mooney. Engineering vascular networks in porous polymer matrices. J. Biomed. Mater. Res. 60:668-678 (2002).

8. M. Simonsand and J. A. Ware. Therapeutic angiogenesis in cardiovascular disease. Nat. Rev., Drug Discov. 2:863-871 (2003).

9. M. C. Peters, B. C. Isenberg, J. A. Rowley, and D. J. Mooney. Release from alginate enhances the biological activity of vascular endothelial growth factor. J. Biomater. Sci., Polym. Ed. 9:1267-1278 (1998).

10. R. Langer. Drug delivery and targeting. Nature 392:5-10 (1998).

11. K. E. Park (ed.) Controlled Drug Delivery: Challenges and Strategies, American Chemical Society, Washington, DC, 1997.

12. W. L. Murphy, M. C. Peters, D. H. Kohn, and D. J. Mooney. Sustained release of vascular endothelial growth factor from mineralized poly(lactide-co-glycolide) scaffolds for tissue engineering. Biomaterials 21:2521-2527 (2000).

13. D. Gilding. Biodegradable Polymers, CRC Press, Boca Raton, FL, 1981.

14. M. H. Sheridan, L. D. Shea, M. C. Peters, and D. J. Mooney. Bioabsorbable polymer scaffolds for tissue engineering capable of sustained growth factor delivery. J. Control. Rel. 64:91-102 (2000).

15. T. P. Richardson, M. C. Peters, A. B. Ennett, and D. J. Mooney. Polymeric system for dual growth factor delivery. Nat. Bio technol. 19:1029-1034 (2001).

16. J. L. Cleland, E. T. Duenas, A. Park, A. Daugherty, J. Kahn, J. Kowalski, and A. Cuthbertson. Development of poly-(D,Llactide-coglycolide) microsphere formulations containing recombinant human vascular endothelial growth factor to promote local angiogenesis. J. Control Rel. 72:13-24 (2001).

17. S. F. Schlossman. Leucocyte Typing V: White Cell Differentiation Antigens: Proceedings of the Fifth International Workshop and Conference held in Boston, USA, 3-7 November, 1993, Oxford University Press, Oxford, NY, 1995.

18. O. Skalli, P. Ropraz, A. Trzeciak, G. Benzonana, D Gillessen, and G. Gabbiani. A monoclonal antibody against alpha-smooth muscle actin: a new probe for smooth muscle differentiation. J. Cell Biol. 103:2787-2796 (1986).

19. L. D. Harris, B. S. Kim, and D. J. Mooney. Open pore biodegradable matrices formed with gas foaming. J. Biomed. Mater. Res. 42:396-402 (1998).

20. A. Rivard, J. E. Fabre, M. Silver, D. Chen, T. Murohara, M. Kearney, M. Magner, T. Asahara, and J. M. Isner. Age- 
dependent impairment of angiogenesis. Circulation 99:111-120 (1999).

21. S. Takeshita, L. P. Zheng, E. Brogi, M. Kearney, L. Q. Pu, S. Bunting, J. F. Symes, and J. M. Isner. Therapeutic angiogenesis. A single intraarterial bolus of vascular endothelial growth factor augments revascularization in a rabbit ischemic hind limb model. J. Clin. Invest. 93:662-670 (1994).

22. S. Takeshita, L. Q. Pu, L. A. Stein, A. D. Sniderman, S. Bunting, N. Ferrara, J. M. Isner, and J. F. Symes. Intramuscular administration of vascular endothelial growth factor induces dosedependent collateral artery augmentation in a rabbit model of chronic limb ischemia. Circulation 90:II228-II234 (1994).

23. B. Witzenbichler, T. Asahara, T. Murohara, M. Silver, I. Spyridopoulos, M. Magner, N. Principe, M. Kearney, J. S. Hu, J. M. Isner Vascular endothelial growth factor-C (VEGF-C/ VEGF-2) promotes angiogenesis in the setting of tissue ischemia. Am. J. Pathol. 153:381-394 (1998).

24. S. Rajagopalan, M. Shah, A. Luciano, R. Crystal, and E. G. Nabel. Adenovirus-mediated gene transfer of VEGF(121) improves lower-extremity endothelial function and flow reserve. Circulation 104:753-755 (2001).

25. J. E. Nor, J. Christensen, D. J. Mooney, and P. J. Polverini. Vascular endothelial growth factor (VEGF)-mediated angiogenesis is associated with enhanced endothelial cell survival and induction of Bcl-2 expression. Am. J. Pathol. 154:375-384 (1999).

26. S. I. Stiver, X. Tan, L. F. Brown, E. T. Hedley-Whyte, and H. F. Dvorak. VEGF-A angiogenesis induces a stable neovasculature in adult murine brain. J. Neuropathol. Exp. Neurol. 63:841-855 (2004).

27. M. Therin, P. Christel, S. Li, H. Garreau, and M. Vert. In vivo degradation of massive poly(alpha-hydroxy acids): validation of in vitro findings. Biomaterials 13:594-600 (1992).

28. A. G. Mikos, Y. Bao, L. G. Cima, D. E. Ingber, J. P Vacanti, and R. Langer. Preparation of poly(glycolic acid) bonded fiber structures for cell attachment and transplantation. J. Biomed. Mater. Res. 27:183-189 (1993).

29. D. J. Mooney, P. M. Kaufmann, K. Sano, K. M. McNamara, J. P. Vacanti, and R. Langer. Transplantation of hepatocytes using porous, biodegradable sponges. Transplant. Proc. 26:3425-3426 (1994).

30. S. L. Ishaug-Riley, G. M. Crane, A. Gurlek, M. J. Miller, A. W. Yasko, and A. G. Mikos. Ectopic bone formation by marrow stromal osteoblast transplantation using poly(DL-lactic-co-glycolic acid) foams implanted into the rat mesentery. J. Biomed. Mater. Res. 36:1-8 (1997).

31. B. S. Kimand and D. J. Mooney. Development of biocompatible synthetic extracellular matrices for tissue engineering. Trends Biotechnol. 16:224-230 (1998).

32. K. Y. Lee, M. C. Peters, K. W. Anderson, and D. J. Mooney. Controlled growth factor release from synthetic extracellular matrices. Nature 408:998-1000 (2000).

33. C. A. Vacanti, R. Langer, B. Schloo, and J. P. Vacanti. Synthetic polymers seeded with chondrocytes provide a template for new cartilage formation. Plast. Reconstr. Surg. 88:753-759 (1991).

34. F. D. Fortuin, P. Vale, D. W. Losordo, J. Symes, G. A. DeLaria, J. J. Tyner, G. L. Schaer, R. March, R. J. Snell, T. D. Henry, J. Van Camp, Van J. J. Lopez, W. Richenbacher, J. M. Isner, R. A. Schatz. One-year follow-up of direct myocardial gene transfer of vascular endothelial growth factor-2 using naked plasmid deoxyribonucleic acid by way of thoracotomy in nooption patients. Am. J. Cardiol. 92:436-439 (2003).

35. E. R. Mohler, III, S. Rajagopalan, J. W. Olin, J. D. Trachtenberg, H. Rasmussen, R. Pak, and R. G. Crystal. Adenoviral-mediated gene transfer of vascular endothelial growth factor in critical limb ischemia: safety results from a phase I trial. Vasc. Med. 8:9-13 (2003).

36. J. A. Nagy, A. M. Dvorak, and H. F. Dvorak. VEGF-A(164/165) and PlGF: roles in angiogenesis and arteriogenesis. Trends Cardiovasc. Med. 13:169-175 (2003). 